\title{
A MIXED BOUNDARY VALUE PROBLEM FOR LAPLACE'S EQUATION INVOLVING A NEARLY CIRCULAR DISK
}

\section{A. ChAKRABARTI and D.P. MANNA}

\author{
Department of Mathematics \\ Indian Institute of Science \\ Bangalore - 560012 \\ India
}

(Received February 20, 1992 and in revised form March 15, 1993)

\begin{abstract}
The electrostatic problem of a nearly circular disk charged to a unit potential is considered for its solution as it serves as the most important and symbolic mixed boundary value problem for Laplace's equation with the aid of which many complicated mixed boundary value problems arising in elasticity and fluid dynamics can be handled for solution. The method used involves the utility of Green's second identity, Abel's integral equations and their inversions, along with a suitably designed perturbation scheme involving the small parameter $\varepsilon(>0)$ occurring in the geometrical representation of the boundary of the nearly circular disk.
\end{abstract}

KEY WORDS AND PHRASES. Mixed boundary value problem, Green's identity, Abel's equation, perturbation scheme.

1991 AMS SUBJECT CLASSIFICATION CODE(S). 31B20.

\section{INTRODUCTION.}

The mixed boundary value problem of solving Laplace's equation

$$
\nabla^{2} v=\frac{\partial^{2} v}{\partial \rho^{2}}+\frac{1}{\rho} \frac{\partial v}{\partial \rho}+\frac{1}{\rho^{2}} \frac{\partial^{2} v}{\partial \theta^{2}}+\frac{\partial^{2} v}{\partial z^{2}}=0
$$

in cylindrical polar co-ordinates $(\rho, \theta, z)$, being satisfied in the half-space $z>0$, under the following boundary conditions

(i) $v=1$ on $z=0$, for $0<\rho<f(\theta) \quad(0 \leq \theta \leq 2 \pi)$

(ii) $\frac{\partial v}{\partial z}=0$ on $z=0$, for $\rho>f(\theta)$

and

(iii) $v, \frac{\partial v}{\partial z} 0$ as $\left(\rho^{2}+z^{2}\right)^{1 / 2} \rightarrow \infty$,

with $f(\theta)$ representing a known differentiable function of $\theta$, for $0 \leq \theta \leq 2 \pi$, serves as a generalization of the corresponding problem in which $f(\theta)=1$, that represents the mixed boundary value problem of determining the axisymmetric electrostatic potential $v(\rho, z)$ (see Sneddon [1]) due to a circular disk of radius unity raised to a unit potential.

The case when

$$
f(\theta)=1-\varepsilon \cos ^{2} \theta \quad(0<\varepsilon \ll 1)
$$


gives rise to a situation in which the disk in question is nearly circular in shape, it being elliptical actually, when $\varepsilon$ is very small as compared to unity.

When such is the shape of the disk, the axisymmetry is lost and it is worthwhile to determine a method by which such an asymmetric mixed boundary value problem for Laplace's equation can be solved, at least approximately, to the order of $\varepsilon$. It so happens that by employing Green's second identity (see [3]) to the function $v(\rho, \theta, z)$ and a suitably chosen Green's function $G\left(\rho, \theta, z ; \rho^{\prime}, \theta^{\prime}, z^{\prime}\right)$ satisfying the equation

$$
\nabla^{2} G=-4 \pi \delta\left(\vec{r}-\vec{r}^{\prime}\right)
$$

and the conditions that

and

(a) $\frac{\partial G}{\partial z}=0$ on $z=0$

(b) $G \rightarrow 0$ as $\left(\rho^{2}+z^{2}\right)^{1 / 2} \rightarrow \infty$,

where $\left.\vec{r}[=(\rho, \theta, z)], \vec{r}^{\prime}\left[=\rho^{\prime}, \theta^{\prime}, z^{\prime}\right)\right]$ represent the position vectors of two distinct points in the halfspace under consideration and $\delta(x)$ represents Dirac's delta function, as explained by R. Shail [2], the problem of solving the equation (1.1) under the conditions (i), (ii), and (iii) can be cast into an integral equation of the first kind for the unknown function $\sigma(\rho, \theta)$ as defined by the relation

$$
\sigma(\rho, \theta)=-\frac{1 \partial v}{2 \pi \partial z}, \text { on } z=0,0<\rho<1-\varepsilon \cos ^{2} \theta,
$$

Then, using the perturbational expansion of $\sigma(\rho, \theta)$ in the form

$$
\sigma(\rho, \theta)=\sigma_{0}(\rho, \theta)+\varepsilon \sigma_{1}(\rho, \theta)+\cdots,
$$

the resulting integral equation is cast into a system of Abel's integral equations after using a Fourier type analysis along with the use of Copson's integral (see Sneddon [1]) formula as given by the relation

$$
\begin{aligned}
& f_{n}\left(\rho, \rho^{\prime}\right)=\frac{1}{2 \pi} \int_{0}^{2 \pi} \frac{\operatorname{cosn}\left(\theta-\theta^{\prime}\right) d \theta}{\left\{\rho^{2}+\rho^{\prime 2}-2 \rho \rho^{\prime} \cos \left(\theta-\theta^{\prime}\right)\right\}^{1 / 2}} \\
= & \frac{2}{\pi} \rho^{-n} \rho^{\prime-n} \int_{0}^{\min \left(\rho, \rho^{\prime}\right)} \frac{t^{2 n} d t}{\left\{\left(\rho^{2}-t^{2}\right)\left(\rho^{\prime 2}-t^{2}\right)\right\}^{1 / 2}}, \cdots
\end{aligned}
$$

Similar boundary value problems, involving boundaries which deviate only a little from the conventional geometrical shapes, have been handled earlier for solution by using a perturbational approach as described above, by several workers (see Happel and Brenner [4] and L. Vijaya Bharathi and A. Chakrabarti [5]).

It is then shown, by using the well-known Abel inversion formulae, that the charge-density on the disk (see Sneddon [1]), i.e., the function $\sigma(\rho, \theta)$ can be expressed in the form

$$
\sigma(\rho, \theta)=\pi^{-2}\left(1-\rho^{2}\right)^{-1 / 2}\left[1+\frac{\varepsilon}{2}\left(1-2 \rho^{2} \cos 2 \theta\right)\right]+\varepsilon \pi^{-2} \rho^{-1} \cos 2 \theta \sec { }^{-1}\left(\rho^{-1}\right),
$$

which reduces to the known result (see Sneddon [1]) for the particular case of a circular disk of unit radius, if we put $\varepsilon=0$.

It may be mentioned here that the axisymmetric problem in the case when $\varepsilon=0$, is amenable to its solution via dual integral equations involving Bessel functions whilst the present asymmetric problem does not enjoy that benefit. 


\section{THE METHOD OF SOLUTION.}

Using Green's second identity to the pair of functions $v\left(\vec{r}^{\prime}\right)$ and $G\left(\vec{r}, \vec{r}^{\prime}\right)$, as explained in the introduction, in the region occupied by the plane $z^{\prime}=0$ and a large semi-spherical surface $\rho^{\prime}=R$ and taking limit as $R \rightarrow \infty$, we obtain the representation for $v(\vec{r})$ as given by the relation

$$
v(\vec{r})=-\frac{1}{4 \pi} \iint_{S} G\left(\vec{r}, \vec{r}^{\prime}\right) \frac{\partial v\left(\vec{r}^{\prime}\right)}{\partial z^{\prime}} d s^{\prime}
$$

where $S$ represents the plane $z^{\prime}=0$.

Choosing

$$
G\left(\vec{r}, \vec{r}^{\prime}\right)=\frac{1}{\left|\vec{r}-\vec{r}^{\prime}\right|}+\frac{1}{\left|\vec{r}+\vec{r}^{\prime}\right|},
$$

which satisfies the relations (1.3) and (1.4), and using the boundary condition (i), we easily derive the following integral equation for the unknown function $\sigma(\rho, \theta)$;

where

$$
\int_{\theta^{\prime}=0}^{2 \pi} \int_{\rho^{\prime}=0}^{\left(1-\varepsilon \cos ^{2} \theta\right)} \sigma\left(\rho^{\prime}, \theta^{\prime}\right) G\left(\rho, \theta, 0 ; \rho^{\prime}, \theta^{\prime}, 0\right) \rho^{\prime} d \rho^{\prime} d \theta^{\prime}=2 ;\left(0<\rho<1-\varepsilon \cos ^{2} \theta\right),
$$

with

$$
\begin{gathered}
\sigma(\rho, \theta)=-\frac{1 \partial v}{2 \pi \partial z} /_{z=0} \\
G\left(\rho, \theta, 0 ; \rho^{\prime}, \theta^{\prime}, 0\right)=\frac{2}{\left\{\rho^{2}+\rho^{\prime 2}-2 \rho \rho^{\prime} \cos \left(\theta-\theta^{\prime}\right)\right\}^{1 / 2}} .
\end{gathered}
$$

Expanding the function $G\left(\rho, \theta, 0 ; \rho^{\prime}, \theta^{\prime}, 0\right)$, as given by the equation (2.5) into the Fourier series

$$
\begin{gathered}
G\left(\rho, \theta, o ; \rho^{\prime}, \theta^{\prime}, 0\right)=2 \sum_{n=0}^{\infty} \lambda_{n} f_{n}\left(\rho, \rho^{\prime}\right) \cos n\left(\theta-\theta^{\prime}\right), \\
\left(\lambda_{0}=1, \lambda_{n}=2, n \geq 1\right)
\end{gathered}
$$

where $f_{n}\left(\rho, \rho^{\prime}\right)$ is as given by the formula (1.7), the integral equation (2.3) takes up the following form

$$
\sum_{n=0}^{\infty} \lambda_{n} \int_{\theta^{\prime}=0}^{2 \pi} d \theta^{\prime} \int_{\rho^{\prime}=0}^{\left(1-\varepsilon \cos ^{2} \theta\right)} \rho^{\prime} \sigma\left(\rho^{\prime}, \theta^{\prime}\right) \cos n\left(\theta-\theta^{\prime}\right) f_{n}\left(\rho, \rho^{\prime}\right) d \rho^{\prime}=1 ;\left(0<\rho<1-\varepsilon \cos ^{2} \theta\right)
$$

Using the perturbational expansion of $\sigma(\rho, \theta)$ in the form (1.6) and expanding the inner integral of equation (2.7) into a Taylor series about $\varepsilon=0$, we obtain, after collecting the various coefficients of powers of $\varepsilon$, that

and

$$
\sum_{n=0}^{\infty} \lambda_{n} \int_{\theta^{\prime}=0}^{2 \pi} d \theta^{\prime} \int_{\rho^{\prime}=0}^{1} \rho^{\prime} \sigma_{0}\left(\rho^{\prime}, \theta^{\prime}\right) \cos n\left(\theta-\theta^{\prime}\right) f_{n}\left(\rho, \rho^{\prime}\right) d \rho^{\prime}=1
$$

$$
\begin{gathered}
\sum_{n=0}^{\infty} \lambda_{n} \int_{\theta^{\prime}=0}^{2 \pi} d \theta^{\prime} \int_{\rho^{\prime}=0}^{1} \rho^{\prime} \sigma_{1}\left(\rho^{\prime}, \theta^{\prime}\right) \cos n\left(\theta-\theta^{\prime}\right) f_{n}\left(\rho, \rho^{\prime}\right) d \rho^{\prime} \\
=\cos ^{2} \theta \sum_{n=0}^{\infty} \lambda_{n} \int_{\theta^{\prime}=0}^{2 \pi} d \theta^{\prime} \int_{\rho^{\prime}=0}^{1} \rho^{\prime} \sigma_{0}\left(\rho^{\prime}, \theta^{\prime}\right) \cos n\left(\theta-\theta^{\prime}\right) f_{n}\left(\rho, \rho^{\prime}\right) d \rho^{\prime},
\end{gathered}
$$

up to the terms of $0(\varepsilon)$.

The Fourier expansion procedure immediately suggest, from (2.8) and (2.9), (using the result 
$\left.\cos ^{2} \theta=\frac{(1+\cos 2 \theta)}{2}\right)$, that we must have that

$$
\sigma_{0}(\rho, \theta)=\sigma_{0}(\rho) \quad \text { (a function of } \rho \text { only) }
$$

and

$$
\sigma_{1}(\rho, \theta)=\sigma_{1}^{(1)}(\rho)+\sigma_{1}^{(2)}(\rho) \cos 2 \theta
$$

where $\sigma_{0}(\rho), \sigma_{1}^{(1)}(\rho)$ and $\sigma_{1}^{(2)}(\rho)$ satisfy the following relations

$$
2 \pi \int_{\rho^{\prime}=0}^{1} \rho^{\prime} \sigma_{0}\left(\rho^{\prime}\right) f_{0}\left(\rho, \rho^{\prime}\right) d \rho^{\prime}=1 \quad(0<\rho<1)
$$

and

$$
\sigma_{1}^{(1)}(\rho)=\frac{1}{2} \sigma_{0}(\rho)
$$

$$
2 \int_{\rho^{\prime}=0}^{1} \rho^{\prime} f_{2}\left(\rho, \rho^{\prime}\right) \sigma_{1}^{(2)}\left(\rho^{\prime}\right) d \rho^{\prime}=\int_{\rho^{\prime}=0}^{1} \rho^{\prime} \sigma_{0}\left(\rho^{\prime}\right) f_{0}\left(\rho, \rho^{\prime}\right) d \rho^{\prime} \quad(0<\rho<1),
$$

Using the result (1.7), and the standard Abel's inversion formulae (see Sneddon [1]), we can easily solve the integral equations (2.12) and (2.14), and we find that

and

$$
\begin{gathered}
\sigma_{0}(\rho)=\pi^{-2}\left(1-\rho^{2}\right)^{-1 / 2} \\
2 \sigma_{1}^{(2)}(\rho)=2 \pi^{-2}\left[\rho^{-1} \sec ^{-1}\left(\rho^{-1}\right)-\rho^{2}\left(1-\rho^{2}\right)^{-1 / 2}\right]
\end{gathered}
$$

Using these results, along with the relations (2.11) and (1.6), finally give the corrected chargedensity $\sigma(\rho, \theta)$ to $0(\varepsilon)$ terms, as given by the relation

$$
\begin{aligned}
\sigma(\rho, \theta)= & \pi^{-2}\left(1-\rho^{2}\right)^{-1 / 2}\left[1+\frac{\varepsilon}{2}\left(1-2 \rho^{2} \cos 2 \theta\right)\right] \\
& +\varepsilon \pi^{-2} \rho^{-1}(\cos 2 \theta) \sec ^{-1}\left(\rho^{-1}\right)
\end{aligned}
$$

which may be regarded as the first order correction to the known result (for $\varepsilon=0$ ) for a charged circular disk in the case when the shape of the boundary of the disk differs very little from a circle. With the aid of this result (2.17), the actual boundary value problem under consideration can be solved by using the relation (2.1), along with the equation (2.2).

It may be mentioned here that even though the mixed boundary value problem under consideration can be attacked for its solution directly by using Galin's theorem (see Sneddon [1]), along with the relations (2.1) and (2.2), the present method of solution appears to be simpler and quicker in the case of a nearly circular disk.

\section{REFERENCES}

1. SNEDDON, I.N., Mixed Boundary Value Problems in Potential Theory, North-Holland, 1966.

2. SHAIL, R., The slow rotation of an axisymmetric solid submerged in a fluid with a surfactant surface layer - I, Int. J. Multiphase Flow 5 (1979), 169-183.

3. ZAUDERER, E., Partial Differential Equations of Applied Mathematics, Wiley Interscience.

4. HAPPEL, J. \& BRENNER, H., Low Reynolds Number Hydrodynamics, Noordhoff, Leyden, 1973.

5. VIJAYA BHARATHI, L. \& CHAKRABARTI, A., Solution of a boundary value problem associated with diffraction of water waves by a nearly vertical barrier, IMA Journal of Applied Mathematics 47 (1991), 23-32. 


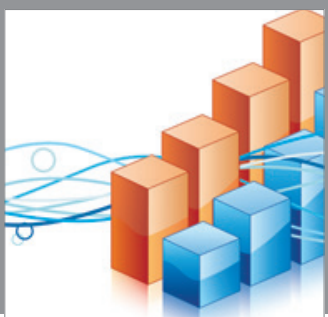

Advances in

Operations Research

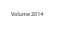

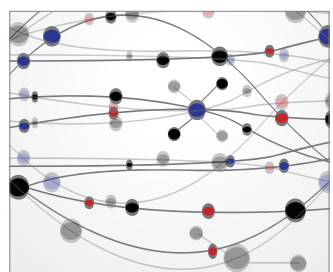

\section{The Scientific} World Journal
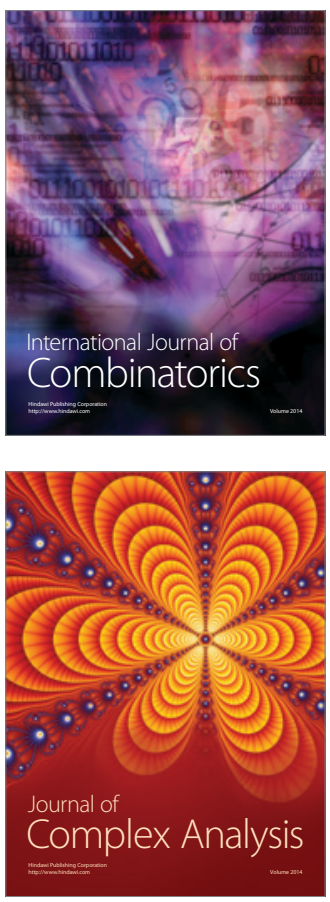

International Journal of

Mathematics and

Mathematical

Sciences
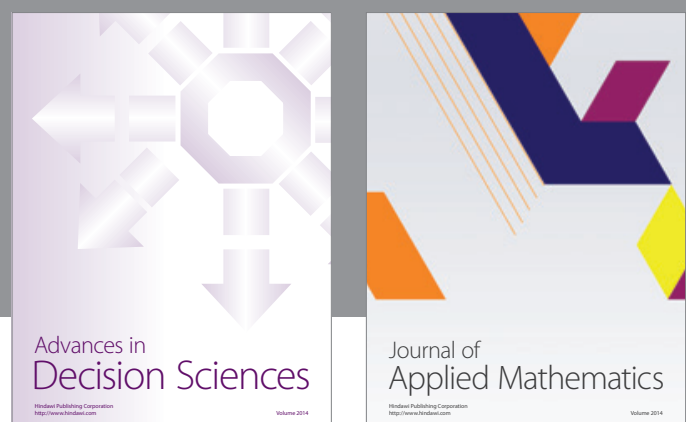

Journal of

Applied Mathematics
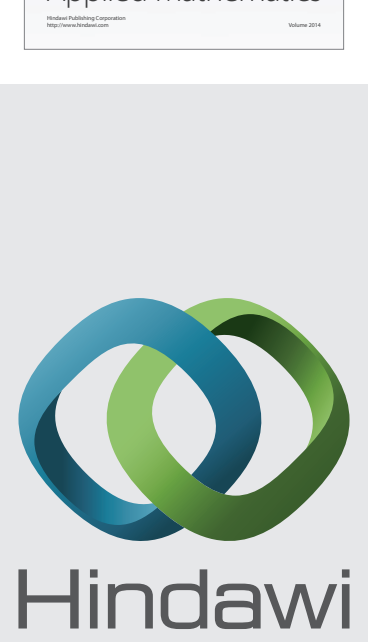

Submit your manuscripts at http://www.hindawi.com
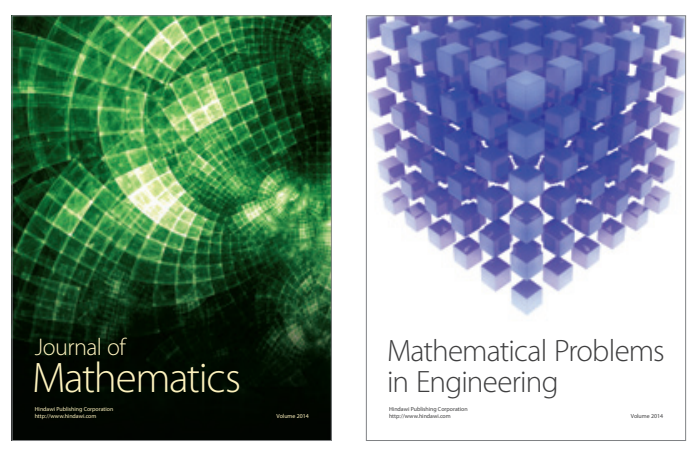

Mathematical Problems in Engineering
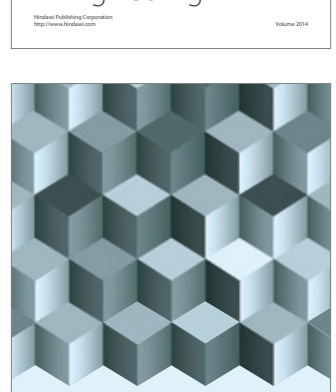

Journal of

Function Spaces
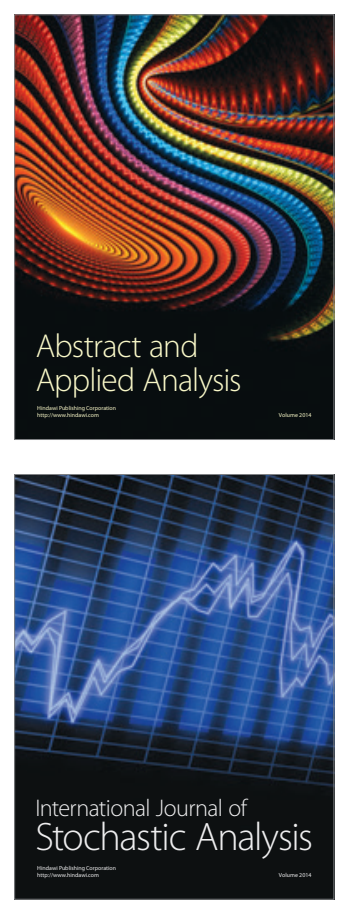

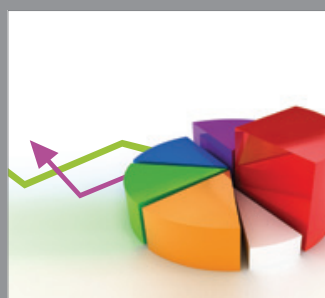

ournal of

Probability and Statistics

Promensencen
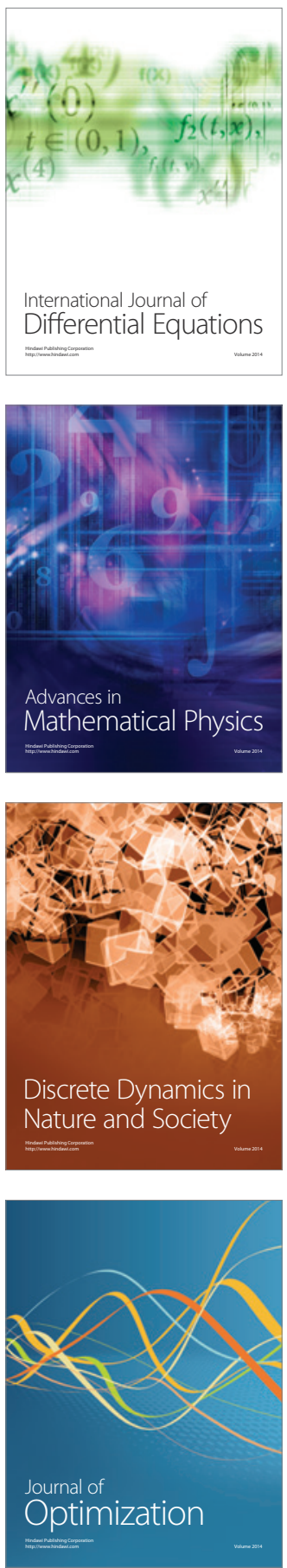